\title{
An Improved DV-Hop Algorithm Based on Shuffled Frog Leaping Algorithm
}

\author{
http://dx.doi.org/10.3991/ijoe.v11i9.5059 \\ Xiaoying Yang, Wanli Zhang, Qixiang Song \\ Suzhou University, Suzhou, China
}

\begin{abstract}
According to that node localization accuracy is not high in the DV Hop localization algorithm, shuffled frog leaping algorithm with many advantages such as the convergence speed is fast, easy to realize and excellent performance of global optimization and so on is introduced into the design of DV-Hop algorithm. A new DV-Hop algorithm based on shuffled frog leaping algorithm (Shuffled Frog Leaping DV-Hop Algorithm, SFLADV-Hop) is proposed in this paper. Based on traditional DV-Hop algorithm, the new algorithm used distance of nodes and position information of anchor nodes to establish objective optimization function and realize the estimation of unknown node coordinate in the final stage of DV-Hop algorithm. The simulation results showed that compared with the traditional DV-Hop algorithm, based on not increasing the sensor node hardware overhead, the improved algorithm can effectively reduce the positioning error.
\end{abstract}

Index Terms-wireless sensor network; DV-Hop algorithm; shuffled frog leaping algorithm; positioning error;

\section{INTRODUCTION}

Node location information plays an important role in wireless sensor network (Wireless Sensor Network, WSN), such as detection target and tracking target, automatic configuring network topology [1]. According to the location whether there is any need for ranging, current localization algorithms are divided into range based and range free two categories [2]. The former has the characteristics of high requirements of the sensor node hardware, but the positioning accuracy is high. The latter has the characteristics of low requirements of the sensor node hardware, low cost, no need for additional hardware, so it is more suitable for large-scale wireless sensor networks in practical application [3].

DV-Hop algorithm is one of the most widely used algorithms in ranging free algorithm, but positioning accuracy of the algorithm is low, so many scholars have made improvements on it. Lu Qingling weighted on average each distance [4]. Li Wenwen used differential evolution algorithm to calculate the location of the unknown node in the localization phase DV-HOP algorithm [5].Lin Jinzhao used the expansion of Taylor series to estimate the location of the unknown node [6].Wang Ying used average network connectivity to improve the hop distance between nodes [7]. Yang Lei set angle threshold to screen anchor nodes [8]. According to the hops of unknown nodes and effects of environmental factors, Feng Jiang weighted the average hop distance [9]. Yang Zhifeng used algebraic reconstruction method to replace the least square method to calculate the coordinate position of unknown nodes [10]. Aiming at the positioning accuracy is not high [11], according to the locating process, combining the basic principles of shuffled frog leaping algorithm, an improved scheme of DV-Hop algorithm based on shuffled frog leaping is proposed in this paper. Compare positioning errors, communication overhead and complexity of the two algorithms under the condition of different node number, different network connectivity, different communication radius and different neighbor anchor nodes through the simulation experiment. The results show that the performance of the improved algorithm is much better than the original one.

\section{DV-HOP ALGORITHM}

\section{A. Positioning process}

Positioning process of DV-Hop algorithm can is divided into three stages.

Step 1: by broadcasting, all nodes obtain minimum hops to each anchor node.

Step 2: By using the coordinate position and number of hops obtained in step 1, the anchor nodes use the formula (1) to calculate the average distance of per hop.

hopsize $_{i}=\frac{\sum_{i \neq j} \sqrt{\left(x_{i}-x_{j}\right)^{2}+\left(y_{i}-y_{j}\right)^{2}}}{\sum_{i \neq j} h_{i j}}$

Where, $x_{i}, y_{i}, x_{j}, y_{j}$ is coordinate position of nodes $i, j$, respectively. $h o p_{i j}$ is the minimum hops between nodes $i, j$.

Step 3: the position coordinates of unknown nodes is calculated by the three sided measurement or the maximum likelihood estimation method.

\section{B. Analysis of positioning error}

After getting distance of three or more anchor nodes, the traditional DV-Hop algorithm uses three sided measurement or the maximum likelihood to estimate coordinate position of the unknown nodes. Coordinate position of each anchor node is, respectively, $\left(\mathrm{x}_{1}, \mathrm{y}_{1}\right),\left(\mathrm{x}_{2}, \mathrm{y}_{2}\right), \cdots,\left(\mathrm{x}_{\mathrm{n}}, \mathrm{y}_{\mathrm{n}}\right)$. Coordinate position of unknown node is $(\mathrm{x}, \mathrm{y})$. The estimated distance between unknown nodes and anchor nodes is, respectively, $\mathrm{d}_{1}, \mathrm{~d}_{2}, \cdots, \mathrm{d}_{\mathrm{n}}$. There are formula (2) set up.

$$
\left\{\begin{array}{c}
\sqrt{\left(x-x_{1}\right)^{2}+\left(x-y_{1}\right)^{2}}=d_{1} \\
\sqrt{\left(x-x_{2}\right)^{2}+\left(x-y_{2}\right)^{2}}=d_{2} \\
\vdots \\
\sqrt{\left(x-x_{n}\right)^{2}+\left(x-y_{n}\right)^{2}}=d_{n}
\end{array}\right.
$$

Linear expression of formula (2) is $\mathrm{AX}=\mathrm{b}$. Where 


$$
\begin{aligned}
X & =(x, y)^{T} \\
A & =\left[\begin{array}{cc}
2\left(x_{1}-x_{n}\right) & 2\left(y_{1}-y_{n}\right) \\
\vdots & \vdots \\
2\left(x_{n-1}-x_{n}\right) & 2\left(y_{n-1}-y_{n}\right)
\end{array}\right], \\
b & =\left[\begin{array}{c}
x_{1}^{2}-x_{n}^{2}+y_{1}^{2}-y_{n}^{2}+d_{n}^{2}-d_{1}^{2} \\
x_{n-1}^{2}-x_{n}^{2}+y_{n-1}^{2}-y_{n}^{2}+d_{n}^{2}-d_{n-1}^{2}
\end{array}\right] .
\end{aligned}
$$

The unknown node coordinate is obtained by using the standard minimum variance estimation, which is $\mathrm{X}^{\mathrm{T}}=\left(\mathrm{A}^{\mathrm{T}} \mathrm{A}\right)^{-1} \mathrm{~A}^{\mathrm{T}} \mathrm{b}$

In step 1 of the traditional DV-Hop algorithm, when getting the number of minimum hop nodes, as long as within the communication radius, both near and far, distance are recorded as 1 jump, which can not reflect the actual distance. Therefore, the average distance per hop which is calculated by using formula (1) always exists error, will inevitably lead to the error accumulation. Thus the coordinate of unknown nodes gotten by formula (2) have larger error, which affects the positioning accuracy of the whole algorithm.

\section{SFLA-DV-HOP ALGORITHM}

\section{A. Shuffled frog leaping algorithm}

Shuffled frog leaping algorithm [12] is a kind of the cooperative co evolutionary algorithm which arose from the natural biological imitation implications. Shuffled frog leaping algorithm simulates process that the frog population exchange information according to the classification of subgroups when search food. Then process is: First, generate a set of initial solutions (population) random from solution space, and then the whole population is divided into a plurality of sub group, subgroup frog perform internal search according to certain search strategies. After ending of search times in internal of subgroup which have been defined, mix all the frogs, and then sort the molecular group, so that the global information can be exchanged between each sub group. Internal search and global information exchange of each subgroup continuously alternate until satisfy the convergence condition or reach the maximum generation so far. The following is a brief introduction to the mathematical model of shuffled frog leaping algorithm [13][14].

\section{(1) subgroup Division}

Set the number of frog in population (candidate solutions) is $N$, the number of subgroups is $k$; the number of candidate solution in population is $\mathrm{n}$. Represents the candidate solution and $D$ represents dimension of candidate solutions. For the initial population which are randomly generated, after descending according to the fitness $f(X)$, the first candidate solution is divided into the first subgroups, the second candidate solution is divided into the second subgroups, $\cdots$, the kth candidate solution is divided into the $k$ th subgroups, the $(k+1)$ th candidate solution is divided into the first subgroups, the $(k+2)$ th candidate solution is divided into the second subgroups, successively repeated until $\mathrm{N}$ candidate solution have been allocated.

\section{(2) Internal search}

Set $X_{b}$ as candidate solutions with the best fitness in a sub group, $X_{w}$ as candidate solutions with the worst fitness in a sub group, $X_{m}$ as candidate solutions with the best fitness in the whole population. Internal search for each subgroup, namely update $X_{w}$ in the sub group, search strategy is shown in formula (3).

$$
X^{\prime}=X_{w}+R \times\left(X_{b}-X_{w}\right)
$$

Where $X^{\prime}$ is a new solution produced by formula (3), $\mathrm{R}$ is a random number between 0 and 1 . If $X^{\prime}$ is better than $X_{w}$, then $X_{m}$ takes replace of $X_{b}$, Repeat the search strategy. Otherwise, $X^{\prime}$ is not better than $X_{w}$, randomly generates a candidate solution to replace $X_{w}$, repeat the above steps, terminate until the search number is greater than the set maximum internal search times.

\section{(3) Global information exchange}

When complete all the subgroups updating, re-execute subgroup division and internal searching, so repeatedly until a termination condition is satisfied (converge to the optimal solution or reach the maximum generation).

\section{B. The SFLADV-Hop algorithm description}

Known from the above analysis, when calculate coordinate of unknown nodes, the estimated value inevitably has big error because of the effects which are environmental factors, communication and so on, thus affecting positioning accuracy of the overall algorithm. Assuming actual distance between unknown node and anchor node is $r_{i}$ and the error is $\varepsilon_{i}$, then meet $\left|r_{i}-d_{i}\right| \leq \varepsilon_{i}$. Then smaller of $\varepsilon_{i}$, the higher of accuracy. When $\varepsilon_{i} \rightarrow 0$, the positioning algorithm obtains the optimum solution. That is, estimating the position is turned into the problem that solves the minimum value, namely function minimization problem. The objective function is taken as:

$$
G=\min \left(\sum_{i=1}^{k}\left(r_{i}-d_{i}\right)\right.
$$

The constraint condition is shown in formula (5).

$$
\left\{\begin{array}{l}
\left|r_{1}-d_{1}\right| \leq \varepsilon_{1} \\
\left|r_{2}-d_{2}\right| \leq \varepsilon_{2} \\
\cdots \\
\left|r_{k}-d_{k}\right| \leq \varepsilon_{k} \\
\sqrt{\left(x-x_{1}\right)^{2}+\left(y-y_{1}\right)^{2}}=d_{1} \\
\sqrt{\left(x-x_{2}\right)^{2}+\left(y-y_{2}\right)^{2}}=d_{2} \\
\cdots \\
\sqrt{\left(x-x_{k}\right)^{2}+\left(y-y_{k}\right)^{2}}=d_{k} \\
r_{k} \geq 0, d_{k} \geq 0, \varepsilon_{k} \geq 0
\end{array}\right.
$$


Shuffled frog leaping algorithm is a swarm intelligence biological evolution algorithm with, which has good flexibility, versatility, fast searching speed and other characteristics and has been widely used in many fields. Thus, the DV-Hop algorithm is improved by combining the shuffled frog leaping in this paper. The shuffled frog leaping algorithm is used to replace the maximum likelihood estimation method to solve coordinate position of the unknown node in the improved algorithm. The specific steps are described as follows.

(1) Define the fitness function of shuffled frog leaping algorithm to measure the quality of solution. The fitness function is selected as the:

$$
f i t=\frac{1}{\alpha+\beta G}
$$

Where $\alpha, \beta$ are constant coefficients of the fitness function, set $\alpha=\beta=1$.

(2) Initialize. Initialize the number of frog individuals as a. The objective function $G$ is viewed as the frog individual. Estimated distance $d_{i}$ is viewed as moving step length of frog individual. The maximum number of iterations is MAXT.

(3) Randomly generates the initial population and calculate the fitness of individuals. Then according to the size of individual fitness values in descending order and assigned to the $\mathrm{s}$ population according to the following principles. The first individual is placed into the first population. The second individual is placed into the second population. $\cdots$, the sth individual is placed into the $s t h$ population. The $(\mathrm{s}+1)$ th individual is placed into the first population. So it has been circulating, until the completion of the distribution of all individuals;

(4) In each population, optimal solutions and the worst solution of the fitness is based on the maximum and minimum value of its fitness function, for $\mathrm{fit}_{b}$ and $\mathrm{fit}_{w}$. And the maximum value $\left(f_{i t}(s)\right)$ of all population is viewed as the global optimal solution of the population ( fit $\left._{b}\right)$.

(6) After internal updating all population, let $\mathrm{t}=\mathrm{t}+1$ and go to step (3), re-divide population and perform the update operation until the maximum number of iterations is reached $M A X T$;

(7)Output the current optimal solution, which is the estimation position of the minimum prediction error;

(8)The algorithm is end.

\section{The flow of SFLADV-Hop algorithm}

To sum up, the flow of SFLADV-Hop algorithm is shown in Figure 1.

\section{SIMULATION EXPERIMENTS}

\section{A. Simulation environment}

In order to compare the performance of SFLADV-Hop algorithm and the traditional DV-Hop algorithm, simulation experiments are carried out on the platform of MATLAB 7.0. The positioning precision of traditional algorithm and the improved algorithm are compared

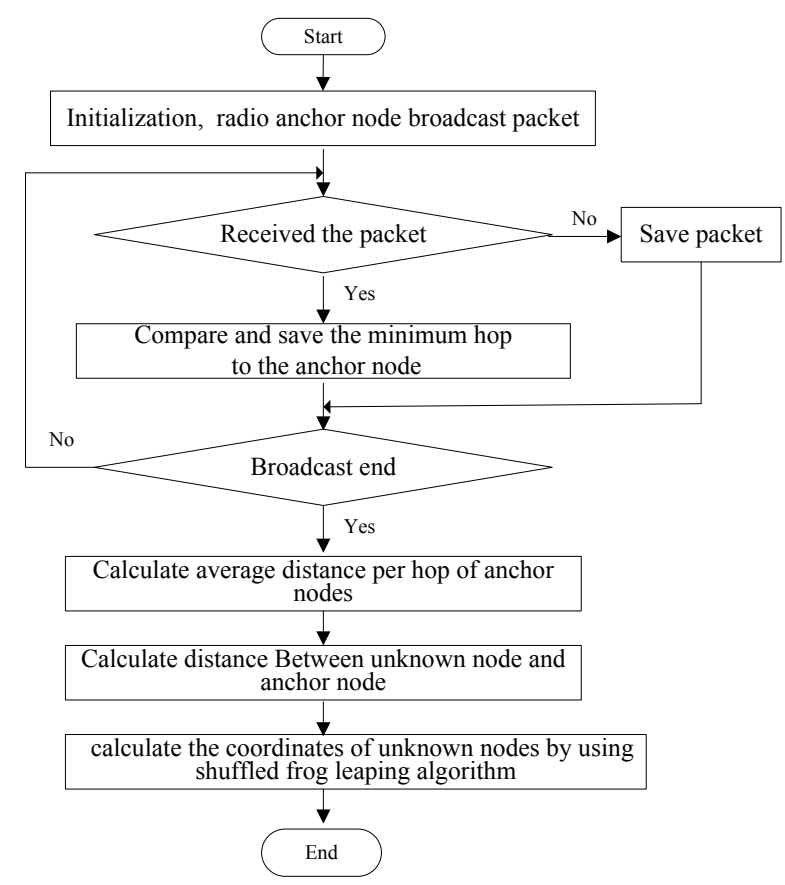

Figure 1. Flow of SFLADV-Hop algorithm

under different proportion of anchor node, different number of nodes and communication radius.

Assuming that nodes are randomly distributed in a square area of $100 \mathrm{~m} * 100 \mathrm{~m}$, randomly selected beacon nodes, all nodes have same radius $(\mathrm{R}=10 \mathrm{~m})$. Using the positioning error as the evaluation indexes of the experiments in this paper, assuming that the real coordinate and estimate coordinates of the unknown node, respectively, is $\left(x_{t}, y_{t}\right),\left(\mathrm{x}_{\mathrm{e}}, \mathrm{y}_{\mathrm{e}}\right)$. Communication radius is $\mathrm{R}$ and positioning error is defined as [15]:

$$
e r r=\sqrt{\left(x_{e}-x_{t}\right)^{2}+\left(y_{e}-y_{t}\right)^{2}} / R
$$

Analyze key factors that influence the SFLA algorithm. The tendency of average positioning error with different frog population number is given, which is shown in Figure 2.

In the initial stage of the simulation, the greater of the number of the Population, the smaller of average positioning error. But at later of simulation, the greater of the number of the Population is, the smaller of average positioning error is. This is because that frog individual performance is generally low at the initial stage, at this time, the greater the number of Population means individual within Population smaller, individual performance update speed will be faster; while at the later of simulation stage, with the overall performance improve, at this time, the smaller the number of population, it can effectively expand the individual optimization goal, to avoid falling into local optimal. According to the simulation results, the number of frog individuals is initialized as 100; a total of 15 Population, the largest number of iterations is 200. In order to eliminate instability of errors caused by node random distribution, in the same network environment, do respectively simulation experiment 100 times and take the average value. 


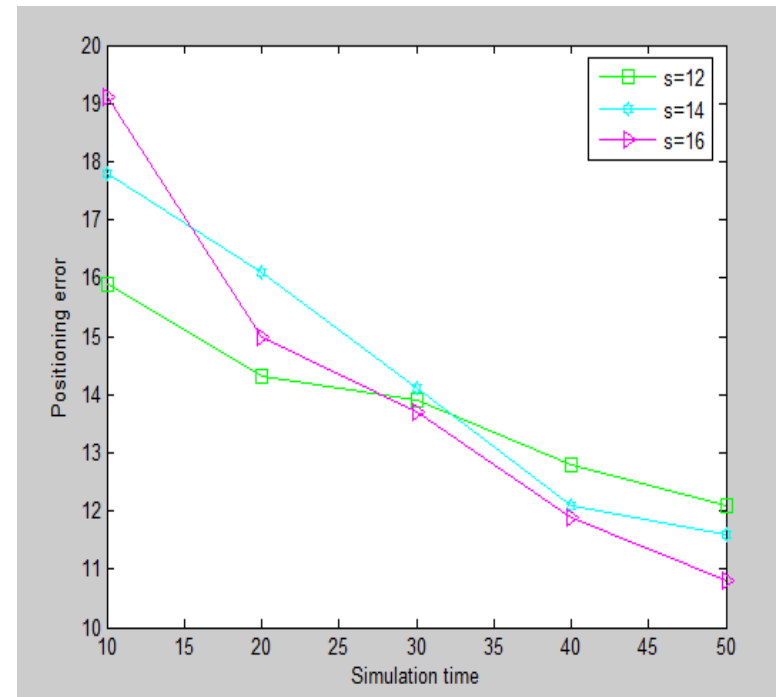

Figure 2. Average positioning error of different number of frog population

\section{B. Analyze simulation results}

(1)Comparison of location result with different number of anchor nodes

To get high precision of positioning, the number of anchor nodes needs enough, but because of the cost factor, the number of anchor nodes is limited in general. In cases that the total number of nodes is 200 , the anchor node number increases from 5 to 40 , compare the performance of SFLADV-Hop algorithm and DV-Hop algorithm, the simulation result is shown in Figure 3.

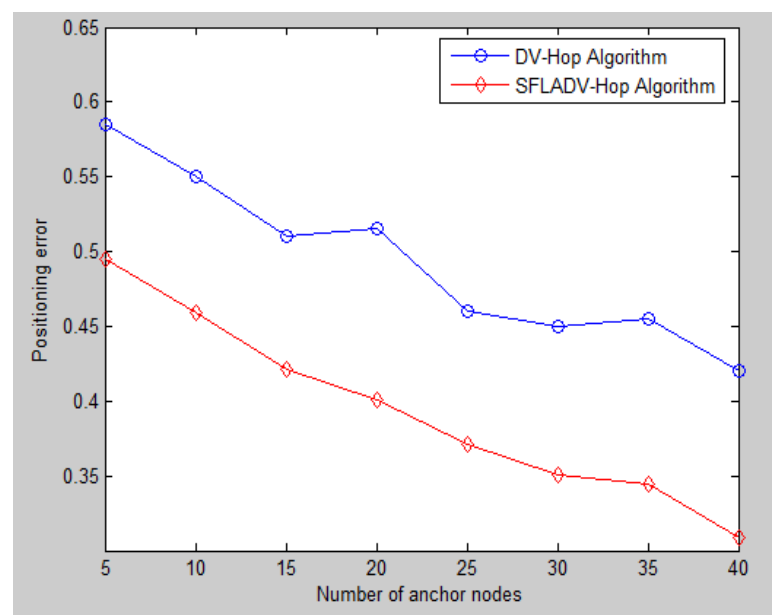

Figure 3. Comparison of positioning error with different anchor nodes

As can be seen from Figure 3, the positioning error of the two algorithms are increased with the number of anchor nodes decreases gradually, but in DV-Hop algorithm, the error is larger as when calculating the coordinate of unknown nodes, the position is no further optimized. While the shuffled frog leaping algorithm is used to estimate coordinates of the unknown nodes in SFLADV-Hop algorithm, so that the position error is lower, closer to the actual position, so the performance of SFLADV-Hop algorithm is significantly better than the traditional DV-Hop algorithm, under the same conditions, compare to the DV-Hop algorithm, the positioning errors of SFLADV-Hop algorithm averagely reduce $11 \% \sim 16 \%$.

(2) Comparison of location result with different number of nodes

Keep the anchor node proportion is $10 \%$ unchanged, change the total number of nodes in the network and compare performance of the EPRDV-Hop algorithm and DV-Hop algorithm. The simulation result is shown in Figure 4.

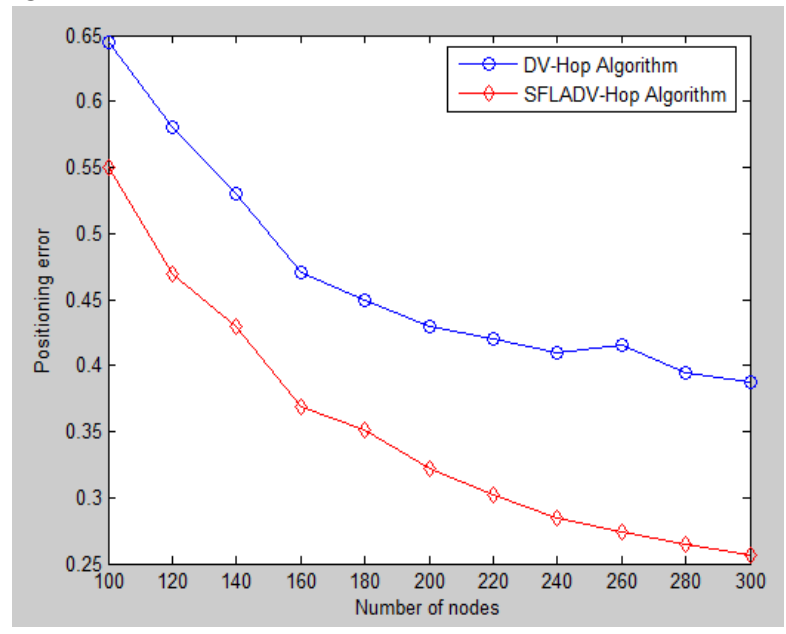

Figure 4. Comparison of positioning error with different number of nodes

As can be seen from Figure 4, the positioning error of the two algorithms decrease gradually as the number of nodes increases, and when the number of nodes is small, SFLADV-Hop algorithm can achieve higher positioning precision, the positioning performance is obviously better than the DV-Hop algorithm, under the same conditions, compare to the DV-Hop algorithm, the positioning errors of SFLADV-Hop algorithm averagely reduce $13 \% \sim 18.5 \%$.

(3) Comparison of location result with different communication radius

Under the condition that keep the number of nodes is 200 , the anchor node proportion is $10 \%$, change the communication radius of nodes. Compare the position performance of two algorithms, the simulation result is shown in Figure 5.

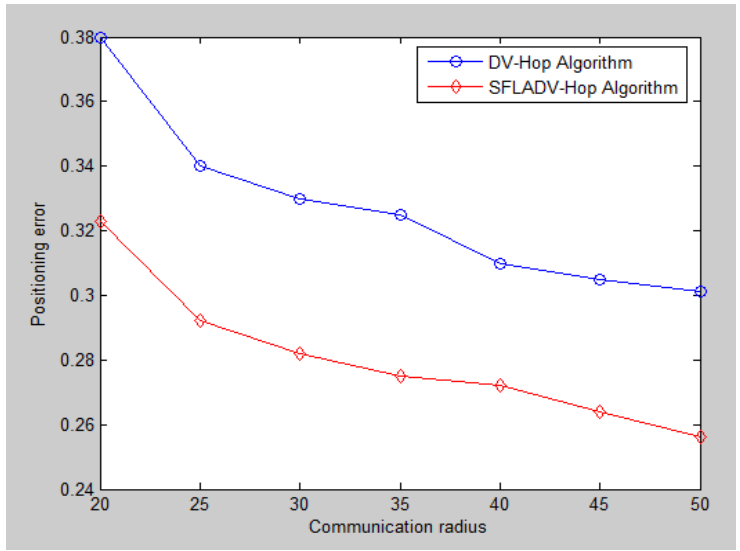

Figure 5. Comparison of positioning error with different communication radius 
As can be seen from Figure 5, with communication radius increasing, communication opportunity between the nodes increase, the positioning error of the two algorithms are gradually reduced. But the positioning error of SFLADV-Hop algorithm is lower than that of DV-Hop algorithm, averagely reduces $11 \% \sim 14 \%$.

\section{CONCLUSION}

Through the analysis of positioning errors caused by the third stage of positioning in DV-Hop algorithm, aiming at the problem that there is much big error in estimating the position of the unknown node by using the maximum likelihood estimation method, an improved algorithm based on shuffled frog leaping algorithm, namely SFLADV-Hop algorithm, is proposed. Shuffled frog leaping algorithm is taken of the maximum likelihood estimation method to estimate the unknown node coordinate in the SFLADV-Hop algorithm. Shuffled frog leaping algorithm with its optimization search ability, makes error between the estimated coordinate and true coordinate of unknown node the minimum, thereby significantly improve the performance of positioning algorithm. Without additional hardware equipment, the SFLADV-Hop algorithm still has the advantage of low cost.

\section{REFERENCES}

[1] Chen T M,Sanchez-Aarnoutse J C,Buford J.Petri netmodeling of cyber-physical attacks on smart grid[J].IEEE Transactions on Smart Grid,2011,2 (4):741-749.

[2] Sun liming, Li Jianzhong, Chen Yu. Wireless sensor network. Beijing: Tsinghua University Press, 2005.

[3] Wu Yu-cheng Li Jiang-wen.Improved DV-Hop Localization Algorithm Based on Optimal Communication Radius of Nodes.Journal of South China University of Technology, Vol. 40, No. 6 June 2012:36-42

[4] Lu Qingling,Bai Mengliang,Zhang Wei,et al. A kind of improved DV-Hop Algorithm[C]//Proc. of the 2nd International Conference on Inteligent Control and Information Processing. Harbin,China:IEEE,Press, 2011:867-869

[5] Li Wenwen,Zhou Wuneng.Genetic algorithm base localization algorithm for wireless sensor network[C]//Proc. of 2011 Seventh International Conference on Natural Computation.Shanghai,China,2011:2096-2099

[6] Lin Jinzhao, Li Xiaolin, Liu Haibo. Improvement and performances of DV hop localization algorithm in wireless sensor networks [J].Journal of Chongqing University,2010,33(2):127-132

[7] Wang Ying, Shi Haoyang. Improved DV-Hop Localization Algorithm for Wireless Sensor Network. Computer Engineering,2012,38(7):66-69

[8] YANG Lei,ZHANG Zheng-bao,XIE Gui-hai,MING Liang, WANG Xin-feng.Improved DV-Hop Positioning Algorithm
Based on Angle Threshold, Computer Engineering,2008,34(20):96-98

[9] FENG Jiang,ZHU Qiang,WU Chun-chun. Research on Improved DV-Hop Localization Algorithm. Computer Engineering,2012,38(19):74-77

[10] YANG Zhi-feng,PEI Teng-da,PEI Bing-nan,CHEN Wei-wei.DVHop Localization Algorithm Based on Algebraic Reconstruction Technique. Computer Engineering,2010,36(15):117-119

[11] LI Yunfei, JIANG Ming, LOU Ke, GE Yuan. Improved DV-Hop localization algorithm in Wireless Sensor Network. Computer Engineering and Applications, 2014, 50 (3): :79-81.

[12] EUSUFF M, LANSEY K E. Optimization of water distribution network design using the shuffled frog leaping algorithm $[\mathrm{J}]$. Water Resources Planning and Management, 2003, 129( 3) : 210 -225 .

[13] JIANG Yubo,ZHAO Pan,QIU Ling.Node Localization Method of Wireless Sensor Network Based on Shuffled Frog Leaping,Journal of Sichuan University of Science \& Engineering( Natural Science Edition),Vol. 27 No. 4,Aug. 2014:34-37

[14] GE Yu,WANG Xue-ping,LIANG Jing.Improved shuffled frog leaping algorithm,Journal of Computer Applications,2012,32(1) : $234-237$

[15] ZHU Min,LIU Hao-lin,ZHANG Zhi-hong,YI Zong-rui.An Improved Localization Algorithm Based on DV-HOP in WSN.JOURNAL OF SICHUAN UNIVERSITY (ENGINEERING SCIENCE EDITION),Vol. 44 No. 1, Jan. 2012:93-98

\section{AUTHORS}

Xiaoying Yang was born in 1983. She received the master degree of computer technology from Guilin University of Electronic Technology in 2010. She is a lecturer at College of Information Engineering, Suzhou University, China. She research interests include Wireless sensor networks and database system. (e-mail: yxiaoying2000@163.com).

Wanli Zhang was born in 1977. He received the master degree of computer technology from Anhui University in 2009. He is a lecturer at College of Information Engineering, Suzhou University, China. He research interests include Wireless sensor networks, Internet of Things and computer network. (e-mail: zhangwanli0557@aliyun.com)

Qixiang Song was born in 1963. He is a professor at College of Information Engineering, Suzhou University, China. He research interests include Wireless sensor networks, Internet of Things and computer network. (szxysqx@163.com)

This work was supported by Young Talents Fund Project in Anhui Province of China (No. 2013SQRL083ZD), Anhui University Provincial Natural Science Research Project (No. KJ2014A247) and University student's Innovative Training Project (201310379019). Submitted 21 September 2015. Published as resubmitted by the authors 20 October 2015. 